\title{
VIABILIDAD DE UNA LINEA DE INVESTIGACION SOBRE LA ENSEÑANZA DE LAS CIENCIAS
}

\section{Humberto Caicedo López*}

\section{Antecedentes}

La línea de investigación aparece en 1982 como resultado de varios años de esfuerzos en el Departamento de Química de la Universidad Pedagógica Nacional por encontrar un camino en la solución del problema de la enseñanza de la química. Habíamos venido trabajando en un programa de investigación que más tarde reconoceríamos como metodológicamente orientado, con resultados más bien descriptivos sin que pudieran ser referidos a principios de educación definidos. Comenzábamos lo que pudiéramos llamar un proyecto de investigación teóricamente orientado (Caicedo, 1989) sustentado en ideas de la sicología y epistemología contemporáneas.

Si bien en una primera etapa las ideas que sustentaban el trabajo de la línea aparecían coherentes y sistemáticamente presentadas, no obstante recogían aproximaciones epistemológicas variadas (Bachelard, Piaget, otros), lo que hicieron que los diseños metodológicos propuestos y los instrumentos no fueran apropiados y confiables. Los resultados de los trabajos en esa visión por consiguiente fueron poco concluyentes.

Una que pudiéramos llamar segunda etapa estuvo influenciada por los trabajos de Viennot (1979), Driver (1986), Gil et al. (1986) y en especial por Posner, et al. (1982) con su Teoría del cambio conceptual. En esta visión constructivista del conocimiento y del aprendizaje se realizaron trabajos con el objeto de explorar "concepciones alternativas" (conceptos no validados por la comunidad científica) que poseen los estudiantes sobre determinado dominio, sus características de persistencia, así como para determinar los efectos que tiene un trabajo pedagógico cuando se diseña para producir cambios conceptuales. En esta ocasión, no obstante tener el respaldo de una teoría más elaborada con claras posibilidades de ser aplicada para abordar problemas de aprendizaje en el aula, los resultados todavía fueron poco discernibles, muy generales, en tal forma que fue difícil hacer deducciones que correspondieran a las preguntas centrales de la línea de investigación.

Un análisis de estos resultados nos mostró que además de que las estrategias utilizadas para propiciar las "acomodaciones" que generaran cambios conceptuales y los instrumentos para que midieran tales cambios no eran los, apropiados, también eran inapropiadas las preguntas claves que originaban los problemas de investigación, tal vez porque nos empeñábamos en hacer cambios drásticos en las concepciones alternativas e ideas intuitivas de los alumnos sin tener en cuenta las características de persistencia y de coherencia que éstas poseen, como lo demuestran numerosos trabajos que se han realizado con este propósito.

\footnotetext{
*Profesor Departamento de Química. Universidad Pedagógica Nacional. Director Centro de Investigaciones U.P.N.
} 
En busca de nuevas alternativas nos encontramos con una serie de trabajos que intentaban resolver problemas de aprendizaje de las ciencias basadas en la Teoría de la Educación de Novak (1978). Esta teoría como la anterior, pertenece al grupo denominado constructivista, que concibe el desarrollo del conocimiento como un proceso de construcción en donde las unidades son los conceptos. Funda su modelo teórico en las concepciones epistemológicas de Kuhn y Toulmín y en las ideas de la sicología cognitiva y del aprendizaje significativo de Ausubel y Novak. Una reinterpretación de estas ideas y la propuesta de estrategias metodológicas guiadas por estos principios es lo que caracteriza el trabajo actual de la línea que ti-ataremos de sintetizar en los apartados siguientes.

\section{Preguntas claves de la línea}

La línea de investigación se ubica dentro de la perspectiva que se denomina de aprendizaje de conceptos. Se adhiere a la concepción de que las personas piensan con conceptos. Que las ciencias son un conjunto de conceptos, principios y teorías, construidos por los científicos junto con procedimientos, instrumentos, técnicas y protocolos de investigación propios. La postura epistemológica subyacente en la línea por consiguiente, no sólo define la comprensión de la naturaleza de la producción del conocimiento sino que orienta además la selección y presentación secuencial de los principios de la disciplina. Supone enfoques de instrucción de tipo constructivista en la que se da importancia a las concepciones y las ideas sobre la ciencia que tienen estudiantes y profesores que, como se ha demostrado, dirigen la forma como se hace la enseñanza, orientan el aprendizaje en el aula y definen los correspondientes procesos de evaluación. La línea en síntesis está comprometida con las ideas de la epistemología constructivista contemporánea y con los principios de la sicología cognitiva sobre el aprendizaje.

Las preguntas centrales que orientan la selección de los problemas, por otra palle, apuntan a esclarecer aspectos relativos a los elementos de la educación: el aprendiz, el maestro, la disciplina, la evaluación y el contexto social, entendido este último como los micro y macroambientes sociales y físicos que influencian los eventos educativos en una cierta circunstancia espacio-temporal.

En esta perspectiva algunas de las preguntas que se han venido estudiando son las siguientes: ¿Qué efecto tiene el conocimiento previo, concepciones alternativas o los conceptos contextualmente erróneos en la construcción de los nuevos conocimientos?; ¿cómo las posturas epistemológicas de estudiantes y profesores orientan la dirección y el estilo de aprendizaje en los primeros y el modelo pedagógico que utilizan los profesores?; ¿son las estrategias pedagógicas de tipo constructivista efectivas para ayudar a los alumnos a comprender la naturaleza y la producción del conocimiento?; ¿qué heurísticas son más efectivas para estimular aprendizajes significativos?; ¿son los cambios de actitud y los cambios metodológicos indicadores de aprendizajes significativos?; ¿qué instrumentos son válidos para medir actitudes y aprendizajes de nivel superior?; ¿qué influencia tiene la organización jerárquica conceptual de los contenidos curriculares en la orientación de aprendizajes significativos?; ¿son los instrumentos metacognitivos, mapa conceptual y diagramas heurísticos y, efectivos para facilitar aprendizajes significativos y la comprensión de la forma como se construye el conocimiento científico?; ¿es la estrategia pedagógica guiada por el constructivismo humano viable para la enseñanza de las ciencias en la escuela?; ¿son los modelos constructivistas opciones viables para acercar al estudiante a la ciencia y la tecnología?; ¿qué modelos pedagógicos emplean los profesores de ciencias y cuál su influencia en el aprendizaje conceptual de la ciencia?; 
¿qué recursos de evaluación son efectivos para medir y estimular aprendizajes significativos?; ¿qué modelos de organización escolar son efectivos para crear climas apropiados para aprendizajes significativos y desarrollo de actitudes positivas hacia la ciencia?

Estas preguntas ya han sido abordadas en una u otra forma en los proyectos de investigación que se han venido desarrollando, algunos ya terminados con informes finales y otros en marcha. El optimismo en la que participamos los miembros del grupo en la obtención de respuestas apropiadas a tales cuestiones radica en que contamos con una teoría con principios definidos que ha venido mostrando potencialidad para ser usada con éxito en el aula de clase a juzgar por los resultados de diversos grupos de investigación. Una interpretación resumida de estos principios, sobre los cuales hemos venido diseñando los proyectos de investigación se dará a continuación.

\section{Bases teóricas y principios guiadores}

La educación se concibe como constituida por experiencias que contribuyen al desarrollo de la persona y le dan una existencia más autónoma. Estas experiencias incluyen eventos de adquisición de conocimientos, eventos que provocan cambios en emociones y sentimientos y mejoras en las actuaciones físicas y motoras. Una experiencia educativa positiva deberá por tanto, mejorar la capacidad de pensar, sentir y actuar del individuo y cambiar, por consiguiente, el significado de sus experiencias. Construir significados es en consecuencia de especial importancia en lo que aquí se entiende por proceso educativo.

La construcción de significados es un proceso activo, es decir, requiere de un esfuerzo individual, consciente. Requiere además, la intervención de otras personas que dotadas de materiales seleccionados con criterio de excelencia, compartan sus significados y guíen la iniciación del proceso de construcción. Se requiere de la enseñanza. Enseñar es, por tanto, compartir deliberadamente los significados para que como resultado cambien también deliberadamente el significado de los eventos, objetos, (experiencia), de los estudiantes.

El papel del maestro es por consiguiente, intercambiar significados y ayudar a dar significado a las experiencias de las personas con las que se interrelaciona como educador. Compartir y negociar significados, sentimientos y acciones que guíen hacia una integración del pensar, sentir y actuar y a un nuevo significado de la experiencia, son eventos educativos que se deben dar entre estudiantes y maestros.

Aprender es otra cosa. Esta es una responsabilidad individual que no se puede compartir y ocurre después de que ha habido posesión de significados. Es individual por cuanto quien aprende aporta sus esquemas o maneras de pensar cuando se enfrenta a la situación de aprendizaje y sus características. La construcción de significados en la nueva situación requiere del aporte de los esquemas mentales del individuo, lo cual hace del aprendizaje un evento educativo de naturaleza idiosincrática.

El aprendizaje significativo (Ausubel, et al., 1986) es un concepto clave en la teoría que desarrolla la línea y es la base para comprender la integración constructiva de pensamientos, sentimientos y acciones que impulsa el desarrollo humano. Como el aprendizaje significativo ocurre cuando la nueva información, los significados compartidos, se relacionan con los esquemas que ya se poseen, es de esperar que para que tal 
aprendizaje ocurra se requiere que el individuo posea conceptos y proposiciones con los cuales pueda relacionarlos consciente y deliberadamente.

Estas relaciones, se hacen alrededor de objetos, eventos, conceptos y principios que constituyen los elementos básicos del conocimiento. Los principios están constituidos por relaciones entre conceptos e indican cómo operan y cómo están estructurados los objetos y eventos. Así, por ejemplo, el principio de incertidumbre y el de la conservación de la masa involucran conceptos y sus relaciones. Indican además como estos conceptos operan para dar significado a las relaciones. En esta visión la orientación en construcción de significados no es una tarea simple y requiere de la comprensión de las implicaciones epistemológicas y sicológicas del evento educativo.

Las aproximaciones epistemológicas de la línea se apartan de las visiones empiropositivistas de la ciencia. Se acercan más bien a la concepción constructivista del desarrollo del conocimiento en donde los conceptos son las unidades de construcción, los principios y teorías de la ciencia son una especie de anteojos a través de los cuales se observan los fenómenos, eventos y objetos y en donde la idea de los conceptos en permanente evolución son la base de la comprensión humana.

Se da la importancia de las ideas de Kuhn ya que el papel que desempeñan los conceptos en el desarrollo de la estructura cognitiva puede ser análogo al papel de los paradigmas kuhnianos en el desarrollo de las ciencias pues si los paradigmas ayudan al científico a interpretar hechos y datos o a buscar información para la solución de los problemas planteados a la ciencia, los conceptos en la estructura cognitiva facilitan el aprendizaje significativo incrementan la capacidad para resolver problemas en áreas específicas del conocimiento. Se acogen también ideas de Toulmin quien utiliza el concepto de evolución para describir la comprensión humana como bases importantes para sustentar las ideas sobre el aprendizaje humano y la construcción de significados.

Se reconoce finalmente que existe una relación íntima entre el aprendizaje significativo y lo que se entiende por construcción del conocimiento. Así, la construcción del conocimiento no es más que una extensión de la capacidad del ser humano para construir nuevos significados, o sea, para construir nuevos conceptos y nuevas relaciones conceptuales en la estructura cognitiva. Construir nuevos conocimientos es así, una forma de aprendizaje significativo y es, por tanto, un fenómeno a la vez sicológico y epistemológico.

Los principios que se tienen en cuenta han sido ya discutidos (Caicedo, 1989) pero en resumen se pueden describir así: tanto los conceptos como las concepciones alternativas se adquieren desde temprana edad y afectan los nuevos aprendizajes. En efecto, desde temprana edad estamos en busca de significados de los eventos y objetos y sus regularidades para luego codificarlos con símbolos o palabras. Estamos construyendo conceptos. En el desarrollo de esta actividad generalmente se construyen ideas e interpretaciones intuitivas sobre los fenómenos de la ciencia que no corresponden a las interpretaciones de la comunidad científica, aparecen lo que se ha denominado esquemas o concepciones alternativas. Estas han demostrado ser resistentes a la modificación y, por el contrario, se refuerzan y aparecen mejor elaboradas por efecto de procesos de instrucción en las que no se tienen en cuenta sus características. Se constituyen en un obstáculo para la comprensión de los conceptos científicos. Por otra parte, también se ha encontrado que cuando se poseen conocimientos previos relevantes a los nuevos conocimientos el resultado es un mayor rendimiento en los procesos de aprendizaje significativo. 
La capacidad de procesamiento de información en el cerebro es limitada, lo cual tiene implicaciones en el aprendizaje de las ciencias sobre todo cuando en la preparación de eventos de aprendizaje se diseñan materiales de instrucción, se asignan tareas y se exigen soluciones a problemas en donde es preciso manipular información que sobrepasa la capacidad mental individual de procesamiento.

El conocimiento se almacena jerárquicamente. Se deduce de la teoría de la asimilación de Ausubel, ya que según éste los nuevos conocimientos se incluyen dentro de conceptos de mayor generalidad (inclusividad) existentes en la estructura cognitiva. Este principio ha dado origen al uso de la técnica del mapa conceptual en la que se representan las relaciones entre los conceptos en forma jerárquica y por consiguiente utilizado como indicador de la organización cognitiva de los conocimientos construidos.

Los compromisos epistemológicos de los estudiantes influyen en el aprendizaje. Las ideas, las concepciones sobre el mundo y la ciencia y su forma de construcción, así como las creencias y los mitos dirigen los procesos de aprendizaje. Estos compromisos forman parte de lo que se ha dado en llamar la ecología conceptual y pueden entrar en conflicto con los principios que plantea una ciencia particular y por consiguiente influyen en los procesos de aprendizaje.

El aprendizaje significativo presupone que el individuo conozca y sea consciente de los procesos cognitivos que intervienen en el aprendizaje y esté dispuesto conscientemente a relacionar en forma sustancial el nuevo material de aprendizaje con lo que posee en su estructura cognitiva. Así mismo, se ha de tener presente en los diseños de eventos educativos que los pensamientos, sentimientos y acciones se integran íntimamente en el individuo y que una estrategia pedagógica constructivista debe estimular para que esta integración ocurra.

\section{El papel de los instrumentos}

En principio se acepta que cualquier metodología, diseño instruccional, entrevista estructurada, no estructurada o clínica y otros procedimientos puede ser susceptible de ser utilizado como instrumento. Lo importante es que éstos tengan coherencia con la formulación teórica. Se hace analogía con la forma de proceder del hombre de ciencia para quien el instrumento es lo que le permite concretar la teoría a un caso particular. El instrumento es en última instancia la teoría misma. Que se pueda observar o que sea lo observable en los eventos educativos depende de la teoría y, por supuesto, del instrumento que lo concrete. Cada instrumento, necesariamente, obedece o se enmarca dentro de un presupuesto teórico o sistema discursivo construido por el educador. Negar esto es caer en el instruccionismo que ha venido guiando la enseñanza de las ciencias.

Dada esta posición frente a los instrumentos, la línea ha venido utilizando el mapa conceptual, el diagrama heurístico $\mathrm{V}$ y la entrevista estructurada de tipo piagetiano, pues se considera que sus fundamentos coinciden con los principios teóricos que la sustentan. El mapa conceptual, tal como lo propone Novak (1985) es un instrumento útil para representar y resumir el sistema de referencia conceptual (los significados) actual de un individuo en un área de conocimiento, como una herramienta para la planificación de las actividades de instrucción y de los contenidos curriculares en forma jerárquica, como instrumento de evaluación y para ayudar a los estudiantes a construir nuevos significados, para aprender a aprender. 
El diagrama heurístico $V$ además de desempeñar papeles similares a los del mapa conceptual, ayuda a maestros y alumnos a profundizar su comprensión sobre la naturaleza del conocimiento científico y sus formas de construcción. En cuanto la entrevista clínica si bien su manejo es más complejo y requiere de entrenamiento experto para su correcta aplicación, se ha encontrado que es la más adecuada cuando se pretende auscultar estructuras cognitivas y compromisos epistemológicos que posee el individuo en un determinado momento.

La experiencia lograda en la utilización de cada uno de estos instrumentos adquiere importancia en la medida que ha ayudado a clarificar la naturaleza del problema de investigación que se adelanta y la de los presupuestos teóricos que guían la aproximación pedagógica utilizada.

\section{Algunos resultados}

Guiados por las preguntas y teniendo en cuenta los elementos claves de la educación, se desarrollaron proyectos cuyos resultados, en los que se muestran datos comparables con aquéllos obtenidos por otros grupos de investigación, nos permitieron reestructurar y hacer ajustes a la línea de investigación, refinar los instrumentos y las metodologías utilizadas.

Los primeros trabajos estuvieron centrados sobre el aprendiz en la auscultación de las ideas intuitivas y concepciones alternativas que poseían al iniciar los estudios formales de ciencias y las variaciones que éstas tenían por efecto de las estrategias utilizadas en el aula de clase. Estudios preliminares, Alarcón (1986), Betancourt (1986), Beltrán (1986), mostraron la existencia de diferentes esquemas construidos intuitivamente sobre los fenómenos naturales y su gran estabilidad.

Un problema que se dio al comienzo fue cómo representar adecuadamente el conocimiento conceptual de los alumnos y los cambios cognitivos ocurridos en el tiempo. Con este propósito se usaron inicialmente formas de entrevista estructuradas aunque no extensivamente, debido a la dificultad de la trascripción y de las técnicas de aplicación y de análisis. Se utilizó luego la técnica del mapeamiento conceptual (Novak y Gowin 1984), como instrumento útil para representar y resumir el sistema de referencia conceptual de un individuo en una determinada área del conocimiento, técnica que más tarde se ampliaría a otras tareas como la de orientar aprendizajes significativos, planeación del currículo y la instrucción y corno instrumento de evaluación.

Trabajos como los de Pérez (1989) y Tovar (1989), utilizan con éxito la técnica del mapa conceptual para explorar concepciones alternativas y/o para estimular aprendizajes significativos. Comprendimos que este podía ser un instrumento que podía ser base para iniciar una serie de proyectos destinados a propiciar aprendizajes conceptuales significativos en el aula de clase. En este propósito se usó complementariamente otra técnica, la $V$ heurística, principalmente para iniciar procesos de construcción de significados en laboratorio.

Otro de los principios que también han sido explorados en estudios sobre el aprendiz es lo que ahora se denomina "ecología conceptual" del individuo el cual supone que dirige el aprendizaje y, por consiguiente, la comprensión de los fenómenos naturales. El trabajo de Torres (1989) basado en esta hipótesis indaga sobre la influencia de las posiciones epistemológicas en el aprendizaje de los conceptos de la química en lo relativo a la teoría del enlace. Los resultados ya definitivos del estudio inducen a concluir que estas 
concepciones afectan la comprensión de los conceptos de la disciplina, sin embargo. no dice mucho sobre cómo superarlos a través de estrategias instruccionales apropiadas. Trabajo adicional debe hacerse en este sentido.

Adicional a lo anterior en esta área todavía quedan por responder otras preguntas: ¿cómo se construyeron los esquemas alternativos y por qué son tan resistentes? ¿Qué procedimientos o estrategias de instrucción pueden utilizarse para producir cambios en tales esquemas? En el primer caso la teoría que sustenta la línea dispone de una hipótesis plausible pero todavía no ha sido explorada, en la segunda, se han iniciado una serie de proyectos en los que principalmente se diseñan estrategias y actividades de instrucción de tipo constructivista, cuyos resultados no comentaremos en este escrito ya que serán objeto de presentaciones especiales posteriores. Estas, sin embargo, tienden en general a producir efectos en los esquemas contextualmente erróneos y a orientar hacia la construcción de nuevos esquemas y nuevos significados esto es, hacia aprendizajes significativos. 


\section{REFERENCIAS BIBLIOGRAFICAS}

ALARCON, et al. (1986). Incidencias de las ideas intuitivas en la aprehensión de conceptos científicos UPN. Bogotá (Trabajo de grado).

AUSUBEL, J. D. et al. (1986). Educational Psycology. A Cognitive View. Holt, Rinehart, Winston, NY.

BELTRAN, et al. (1986). Incidencias de las nociones de calor, trabajo y energía en la aprehensión del concepto de Entalpía. UPN. Bogotá. (Trabajo de grado).

BETANCOURT, et al. (1986). Problemas en la aprehensión de los conceptos Punto de fusión y Punto de ebullición. UPN. Bogotá. (Trabajo de grado).

CAICEDO, H. (1989). Tendencias en la investigación sobre la enseñanza de las ciencias. Educación y Cultura. Nos. 19, 11-15.

DRIVER, R. (1986). Psicología Cognitiva y Esquemas Conceptuales de los alumnos. Enseñanza de las ciencias.

GIL. D. (1986). La metodología científica y la enseñanza de las ciencias. Enseñanza de las Ciencias. V. 4 No. 2, 11-121.

NOVAK, J. D. (1978). A theory of Education. Cornell University Press.

NOVAK, J. D. (1984). Learnig How to Learn. Cambridge University Press.

PEREZ, R. (1989). Aprendizaje de conceptos Acido Base UPN. Bogotá (Tesis).

POSNER, et al. (1982). Accomodation of a Scientific Conception. Toward a Theory of Conceptual Change. Sc. Educ. 66(2) 211-277.

TORRES, D. (1989). Aplicación de la TCC al aprendizaje de conceptos en química. UPN. Bogotá. (Tesis).

TOVAR, G. (1989). Aplicación de la teoría de Novak al aprendizaje de conceptos en química. UPN. Bogotá. (Tesis).

VIENNOT. L. (1979). Spontaneus Reasoning in Elementary Dynamics. Eur. J. Sc. Educ. 